\title{
Microhook ab interno trabeculotomy, a novel minimally invasive glaucoma surgery
}

This article was published in the following Dove Press journal:

Clinical Ophthalmology

\author{
Masaki Tanito \\ Division of Ophthalmology, Matsue \\ Red Cross Hospital, Matsue, Japan
}

\begin{abstract}
Trabeculotomy (LOT) is performed to reduce the intraocular pressure in patients with glaucoma, both in children and adults. It relieves the resistance to aqueous flow by cleaving the trabecular meshwork and the inner walls of Schlemm's canal. Microhook ab interno LOT ( $\mu$ LOT), a novel minimally invasive glaucoma surgery, incises trabecular meshwork using small hooks that are inserted through corneal side ports. An initial case series reported that both $\mu$ LOT alone and combination of $\mu$ LOT and cataract surgery normalize the intraocular pressure during the early postoperative period in Japanese patients with glaucoma. Microhook can incise the inner wall of Schlemm's canal without damaging its outer wall easier than the regular straight knife that is used during goniotomy. Advantages of $\mu$ LOT include: a wider extent of LOT (two-thirds of the circumference), a simpler surgical technique, being less invasiveness to the ocular surface, a shorter surgical time than traditional ab externo LOT, and no requirement for expensive devices. In this paper, the surgical technique of $\mu$ LOT and tips of the technique are introduced.
\end{abstract}

Keywords: trabecular meshwork, Schlemm's canal, intraocular pressure, surgical procedure, glaucoma

\section{Introduction}

Trabeculotomy (LOT) is performed to reduce the intraocular pressure (IOP) in patients with glaucoma, both children and adults. It relieves the resistance to aqueous flow by cleaving the trabecular meshwork and the inner walls of Schlemm's canal. ${ }^{1-4}$ Because there is no bleb involved in decreasing the IOP reduction, there is less likelihood of LOT causing vision-threatening complications, for example, flat anterior chamber (AC), bleb leaks, hypotony maculopathy, choroidal detachment, and bleb infections that can occur after trabeculectomy performed with antifibrotic agents. ${ }^{1,5}$

The ab externo approach has been used to perform LOT in combination with metal trabeculotomes that incise one-third of the meshwork, ${ }^{1-4}$ or with 5-0 and 6-0 polypropylene sutures and a microcatheter that incise the full $360^{\circ}$ of the meshwork. ${ }^{6-8}$ More recently, surgeons also have reported LOT techniques that are used with the ab interno approaches. ${ }^{9,10}$ My colleagues and I initially reported the case of both eyes of 1 patient with steroid-induced glaucoma who underwent a novel ab interno LOT, which we referred to as microhook LOT ( $\mu \mathrm{LOT}$ ) (Figure 1A-C). ${ }^{11}$ Because of the substantial IOP decrease in that case and less ocular surface invasiveness, we began to perform the procedure in other cases.

In this commentary paper for our previous publications, ${ }^{11-14} \mathrm{I}$ introduce the surgical technique of $\mu \mathrm{LOT}$ and tips of the technique. 

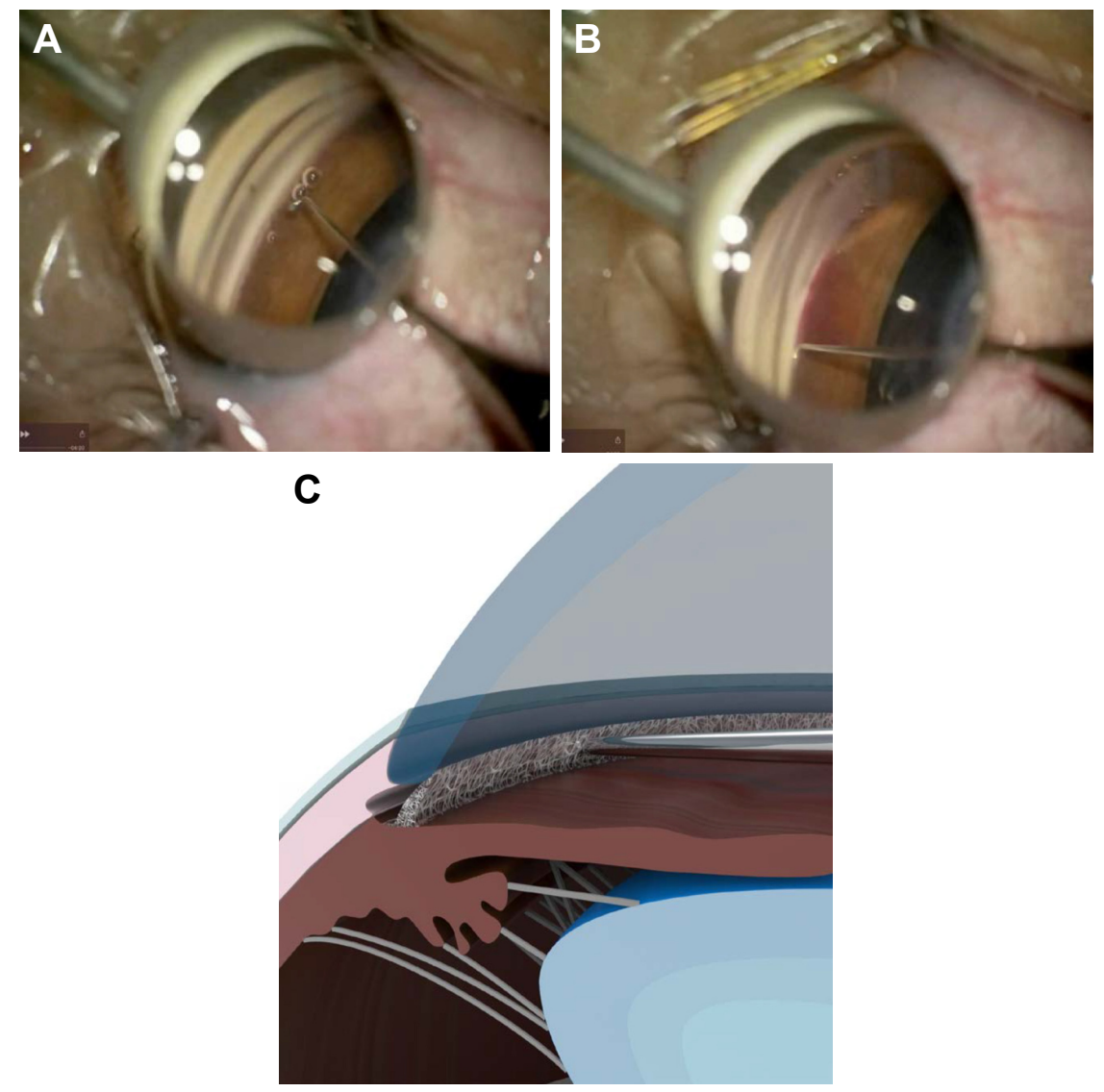

Figure I Intraoperative findings of microhook ab interno trabeculotomy.

Notes: (A) With the temporal angle observed using a Swan-Jacob gonioprism lens, an angled microhook is inserted into the anterior chamber through the corneal incision at the nasal position. (B) The tip of the microhook is inserted directly into the Schlemm's canal and moved circumferentially to incise the inner wall of the Schlemm's canal and trabecular meshwork. (C) Schematic drawing of meshwork incision by the tip of the hook. Courtesy of Inami Co. Ltd.

\section{Surgical technique}

With the technique described in this paper, $\mu$ LOT incises the trabecular meshwork up to two-thirds of the circumference in total (Figure 2A); this is wider than the traditional ab externo LOT (Figure 2B). During $\mu$ LOT surgery, 3 types of microhooks, that is, straight (M-2215S, Figure 3A), angledright (M-2215R, Figure 3B), and angled-left (M-2215L, Figure 3B), are used. For operability, a straight hook is used to incise the nasal angle (Figure 3A) and the right-angled and left-angled hooks are used to incise the temporal angle (Figure 3B). Currently, these hooks can be obtained from the manufacturer (Inami \& Co., Ltd, Tokyo, Japan) in Japan. Standard sub-tenon anesthesia using $2 \%$ lidocaine or intracameral anesthesia using $1 \%$ lidocaine is induced before the procedure. Viscoelastic material (1\% sodium hyaluronate, Opegan Hi, Santen Pharmaceutical, Osaka, Japan) is injected into the $\mathrm{AC}$ through the clear corneal ports created using a 20-gauge micro-vitreoretinal (MVR) knife (Mani, Utsunomiya, Japan) at the 2-3 and 9-10 o'clock positions. Using a Swan-Jacob gonioprism lens (Ocular Instruments, Bellevue, WA, USA) to observe the angle opposite from the corneal port, a microhook is inserted into the $\mathrm{AC}$ through the corneal port. The tip of the microhook is then inserted into the Schlemm's canal and moved circumferentially to incise the inner wall of the Schlemm's canal and trabecular
A

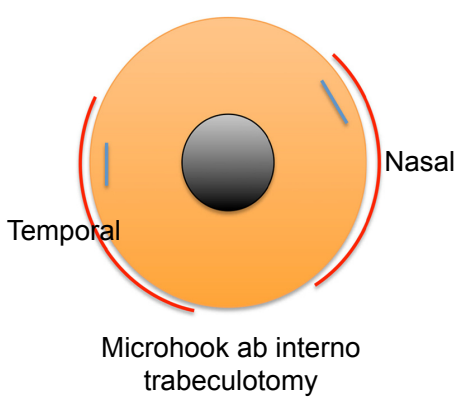

B

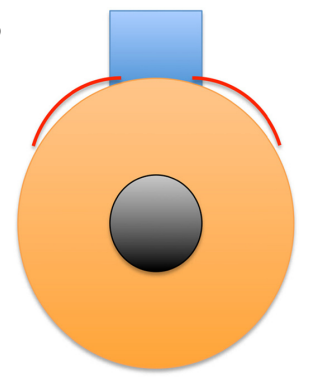

Ab externo trabeculotomy
Figure 2 Typical extent of incision in microhook ab interno trabeculotomy and traditional ab externo trabeculotomy.

Notes: (A) Microhook ab interno trabeculotomy incises trabecular meshwork one-third of the circumference in each of nasal and temporal sides, resulting in the incision extending to two-thirds of the circumference in total. (B) Traditional ab externo trabeculotomy incises trabecular meshwork between a one-quarter and one-third of the circumference when the trabeculotomes are used on both sides of the scleral flap. Red arcades indicate the extent of trabeculotomy, blue lines indicate the corneal side port, and blue rectangle indicates the scleral flap. 
A
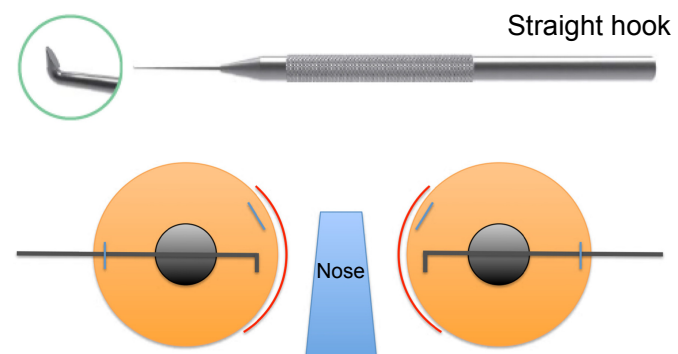

Right eye

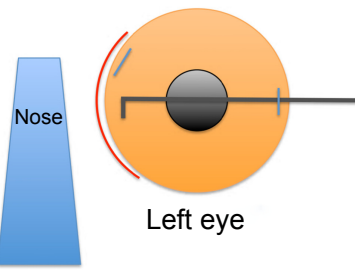

B

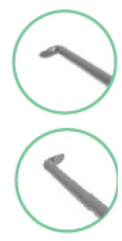

Angled-left hook

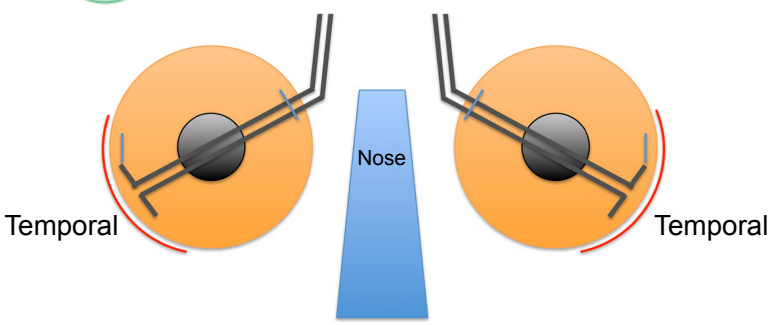

Figure 3 There types of microhooks and schematic drawings of their use.

Notes: (A) A straight hook (M-22I5S) is designed to be used for incising the nasal angle. The hook is inserted into the anterior chamber through the corneal incision at the temporal position. (B) Angled-right (M-22I5R) and angled-left (M-22I5L) hooks are designed to be used for incising the temporal angle. These hooks are inserted into the anterior chamber through the corneal incision at the nasal position. Red arcades indicate the extent of trabeculotomy and blue lines indicate the corneal side port.

meshwork over 3 clock hours. Using the same procedure, LOT is performed in the opposite angle using a microhook that is inserted through another corneal port. After the viscoelastic material is aspirated, the corneal ports are closed by corneal stromal hydration. At the end of surgery, $1.65 \mathrm{mg}$ of dexamethasone sodium phosphate (Decadron, Aspen Japan, Tokyo, Japan) is injected subconjunctivally and $0.3 \%$ ofloxacin ointment (Tarivid, Santen Pharmaceutical) is applied. Finally, 1.5\% levofloxacin (Nipro, Osaka, Japan) and 0.1\% betamethasone (Sanbetason, Santen Pharmaceutical) are applied topically 4 times daily for 3-4 weeks postoperatively in all cases. When simultaneous cataract surgery is required, I usually do small incisional cataract surgery before performing $\mu$ LOT through a 2.2-mm-wide clear corneal incision created at the 9-10 o'clock position (ie, temporal incision for the right eye and nasal incision for the left eye). The video of the surgery performed with the technique described in this paper is available on the Internet (see video online; https:// www.youtube.com/watch?v=VPWhsEsfrn8).

\section{Tips of the technique}

For good visualization of angle structure, proper positioning of the patient's head and surgical microscope is important. Figures 4A and 4B show the regular position of patient's head and microscope. For approaching the temporal angle, I tilt the patient's head to the temporal side, and turn the microscope toward the nasal side (Figure 4C and D). After that, for approaching nasal angle, I tilt the patient's head to the nasal side, and turn the microscope toward the temporal side (Figure 4E and F). In the initial case report, I used a regular straight Sinskey hook to incise the meshwork. ${ }^{12}$ Because the tip of a regular Sinskey hook is dull, I had to create a small goniotomy using an MVR knife before
I insert the tip of the hook into the Schlemm's canal. By sharpening the tip into a spatula shape, the microhook used currently can be inserted directly into the Schlemm's canal without the need to create an entry site, which results in a simplified surgical technique and shorter surgical time. To minimize damage to the outer wall of the Schlemm's canal while inserting the tip of the hook into the Schlemm's canal, I recommend perforating the meshwork by gently sliding, rather than stabbing, the tip into the meshwork surface. After insertion, depth of the tip can be monitored by visualizing the tip through the trabecular meshwork; the inability to visualize the tip through the meshwork usually indicates that the tip has been inserted too deep. With adequate direction and depth, only minor resistance is felt when moving the tip circumferentially; any resistance usually indicates that the tip has been inserted too deep. To avoid unintended tissue damage around the trabecular meshwork, the correct insertion, direction, and depth of the tip of the hook should be monitored carefully during the procedure. Proper opening of the trabecular meshwork can be observed using microscope-integrated optical coherence tomography (OCT) (RESCAN 700, Carl Zeiss Meditec Japan, Tokyo, Japan) in combination with a gonioprism ${ }^{12}$ intra-operatively or by anterior-segment OCT (Casia 2, Tomey Corporation, Nagoya, Japan) post-operatively. ${ }^{14}$

\section{Ethics approval}

All procedures followed were in accordance with the ethical standards of the responsible committee on human experimentation (institutional and national) and with the Helsinki Declaration of 1964, as revised in 2013. Written informed consent was obtained from all patients for being included in the study. This manuscript includes text extracts from my 

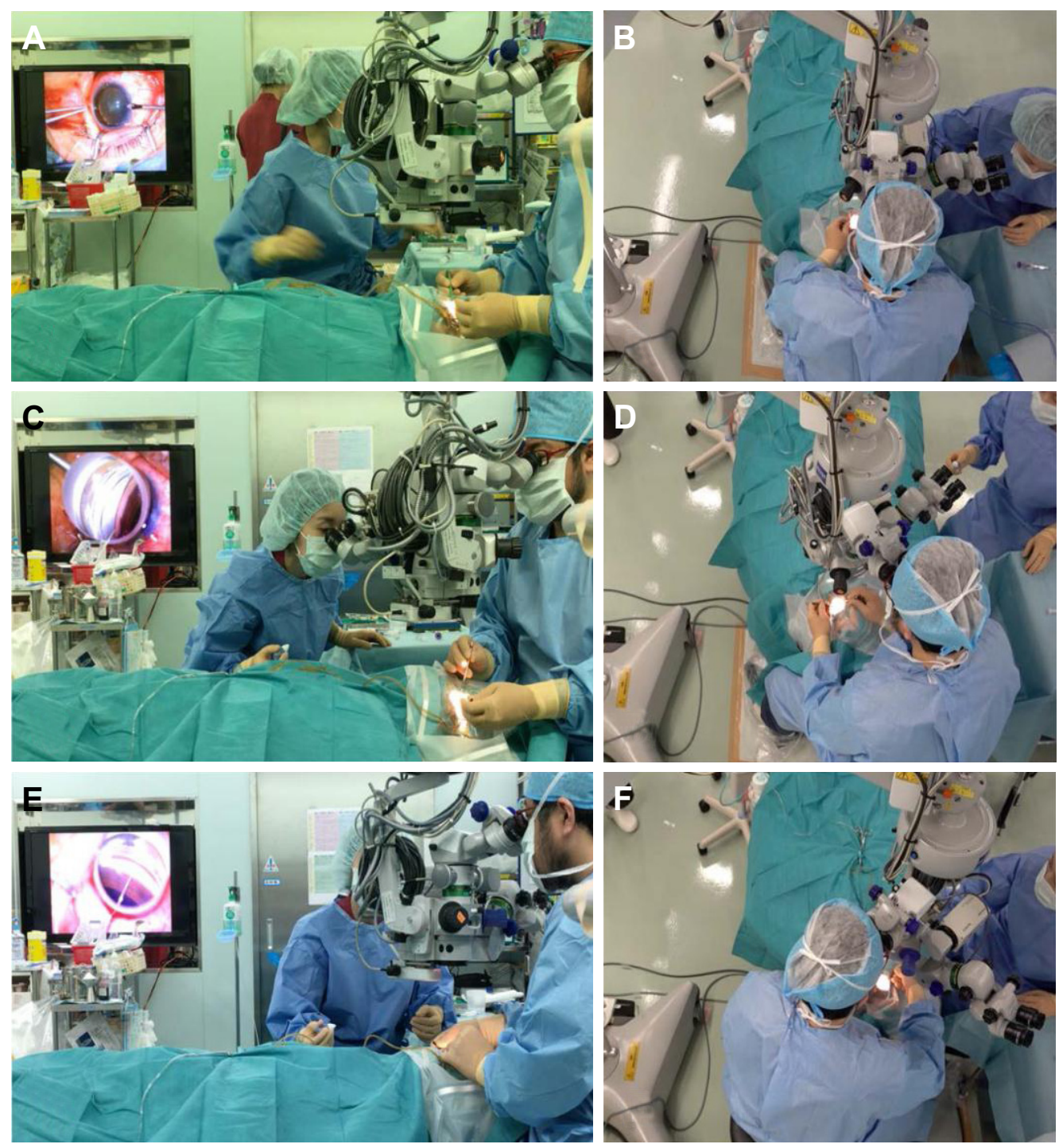

Figure 4 Side view (A, C, E) and overhead view (B, D, F) of positions of the patient's head and surgical microscope during microhook ab interno trabeculotomy in the left eye.

Notes: $(\mathbf{A})$ and (B) show regular positions of the patient's head and microscope. (C) and (D) show that, for approaching the temporal angle, the patient's head is tilted to the temporal side, and the microscope is turned towards the nasal side. (E and $\mathbf{F}$ ) show that, for approaching the nasal angle, the patient's head is tilted to the nasal side, and the microscope is turned towards the temporal side.

previous publications ${ }^{13}$ that were used with permission of Japanese Ophthalmological Society.

\section{Discussion}

In the initial case series, $\mu \mathrm{LOT}$ alone decreased the IOP from the preoperative value of 25.9 to $14.7 \mathrm{mmHg}$, a $43 \%$ decrease at the final 6-month evaluation, ${ }^{12}$ and $\mu$ LOT combined with cataract surgery decreased the IOP from the preoperative value of 16.4 to $11.8 \mathrm{mmHg}$, a $28 \%$ decrease at the final 9.5-month evaluation. ${ }^{13}$ In this case series, hyphema with niveau formation and hyphema washout were the most common postoperative complications and intervention, respectively. ${ }^{12,13}$ Transient IOP spike, cataract progression, vitreous hemorrhage, and macular edema were the other complications reported. ${ }^{12,13}$ Although most of the surgical complications are relatively minor and resolve spontaneously, the decision to perform $\mu \mathrm{LOT}$ should be carefully considered in glaucomatous eyes with advanced visual field defects because post-surgical IOP spike can be related to the decrease in visual acuity. ${ }^{13}$

Incision of the inner wall without damaging the outer wall of the Schlemm's canal can sometimes be difficult when using a straight knife (ie, goniotomy); therefore, $\mu$ LOT seems to be an easier procedure than goniotomy. Conjunctival and scleral sparing with the ab interno technique, short surgical time ( $<10$ minutes), moderate IOP reduction, and no bleb-related complications fulfill the conditions of minimally invasive glaucoma surgery, ${ }^{15,16}$ as happens with the recent techniques 

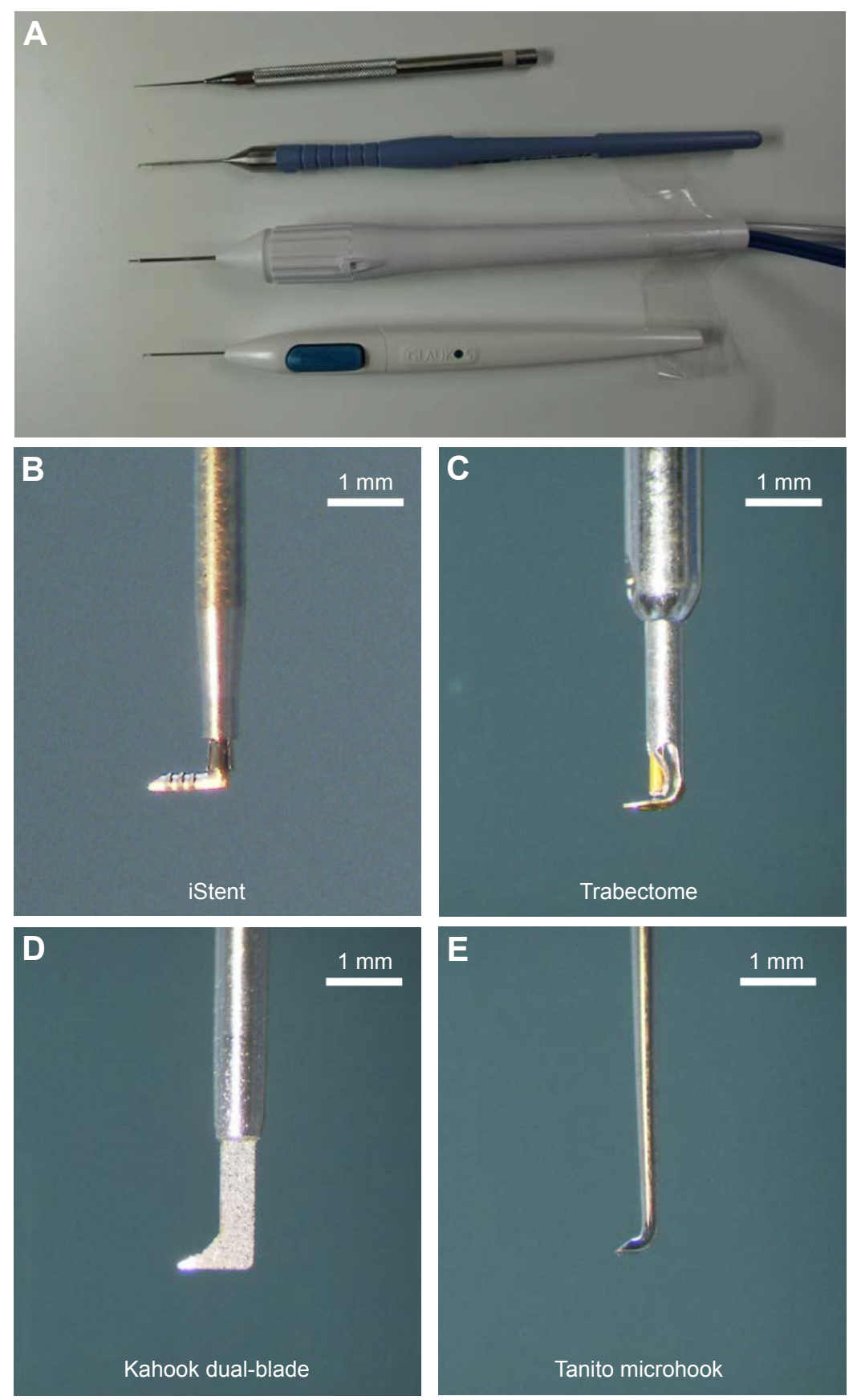

Figure 5 Handpieces (A) and tips of the handpieces (B-E) of various gonio-surgery devices.

of ab interno LOT/trabeculectomy and gonio-bypass surgeries such as the Trabectome (NeoMedix Corp., Tustin, CA, USA), ${ }^{17}$ iStent (Glaukos, Laguna Hills, CA, USA), ${ }^{18}$ gonioscopy-assisted transluminal LOT, ${ }^{9,10}$ canaloplasty, ${ }^{19,20}$ dual-blade trabeculectomy (New World Medical Inc., Rancho Cucamonga, CA, USA), ${ }^{21,22}$ and TRAB360 surgery (Sight Sciences Inc., Menlo Park, CA, USA). Because the tip of the hook is much smaller than the other gonio-surgery devices (Figure 5), to me, intracameral manipulation of the hook is easier than the other devices. Another advantage of $\mu \mathrm{LOT}$ is that expensive devices are not needed. A perfusion study of autopsy eyes reported that incisions in the trabecular meshwork for 1, 4, and 12 o'clock hours eliminated 30\%, $44 \%$, and $51 \%$, respectively, of outflow resistance, at the perfusion pressure of $7 \mathrm{mmHg}$, and $30 \%, 56 \%$, and $72 \%$, respectively, of outflow resistance at the perfusion pressure of $25 \mathrm{mmHg},{ }^{23}$ indicating that wider extent of LOT with $\mu \mathrm{LOT}$ than other surgeries such as traditional ab externo LOT, trabecutome, and dual-blade trabeculectomy can be another advantage, but this requires testing in future comparative 
studies for these procedures. I believe that the $\mu$ LOT is worth further evaluation in a larger and longer longitudinal study or in a comparative study of other surgeries such as ab externo LOT and other trabecular meshwork surgeries.

\section{Acknowledgments}

The microhooks used in this study are co-developed by Masaki Tanito and Inami \& Co., Ltd (Tokyo, Japan), and are provided by Inami \& Co., Ltd.

\section{Author contributions}

The named author meets the International Committee of Medical Journal Editors (ICMJE) criteria for authorship for this manuscript, takes responsibility for the integrity of the work as a whole, and has given final approval to the version to be published.

\section{Disclosure}

Masaki Tanito receives a royalty from Inami \& Co., Ltd (Tokyo, Japan). The author reports no other conflicts of interest in this work.

\section{References}

1. Chihara E, Nishida A, Kodo M, et al. Trabeculotomy ab externo: an alternative treatment in adult patients with primary open-angle glaucoma. Ophthalmic Surg. 1993;24(11):735-739.

2. Tanihara H, Negi A, Akimoto M, et al. Surgical effects of trabeculotomy ab externo on adult eyes with primary open angle glaucoma and pseudoexfoliation syndrome. Arch Ophthalmol. 1993;111(12):1653-1661.

3. Tanito M, Ohira A, Chihara E. Surgical outcome of combined trabeculotomy and cataract surgery. J Glaucoma. 2001;10(4):302-308.

4. Tanito M, Ohira A, Chihara E. Factors leading to reduced intraocular pressure after combined trabeculotomy and cataract surgery. J Glaucoma. 2002;11(1):3-9.

5. Kashiwagi K, Kogure S, Mabuchi F, et al; Collaborative Bleb-Related Infection Incidence and Treatment Study Group. Change in visual acuity and associated risk factors after trabeculectomy with adjunctive mitomycin C. Acta Ophthalmol. 2016;94(7):e561-e570.

6. Beck AD, Lynch MG. 360 degrees trabeculotomy for primary congenital glaucoma. Arch Ophthalmol. 1995;113(9):1200-1202.

7. Chin S, Nitta T, Shinmei Y, et al. Reduction of intraocular pressure using a modified 360-degree suture trabeculotomy technique in primary and secondary open-angle glaucoma: a pilot study. J Glaucoma. 2012; 21(6):401-407.
8. Dao JB, Sarkisian SR Jr, Freedman SF. Illuminated microcatheterfacilitated 360-degree trabeculotomy for refractory aphakic and juvenile open-angle glaucoma. J Glaucoma. 2014;23(7):449-454.

9. Grover DS, Godfrey DG, Smith O, Feuer WJ, Montes de Oca I, Fellman RL. Gonioscopy-assisted transluminal trabeculotomy, ab interno trabeculotomy: technique report and preliminary results. Ophthalmology. 2014;121(4):855-861.

10. Sato T, Hirata A, Mizoguchi T. Prospective, noncomparative, nonrandomized case study of short-term outcomes of 360 degrees suture trabeculotomy ab interno in patients with open-angle glaucoma. Clin Ophthalmol. 2015;9:63-68.

11. Tanito M, Sano I, Ikeda Y, Fujihara E. Microhook ab interno trabeculotomy, a novel minimally invasive glaucoma surgery, in eyes with open-angle glaucoma with scleral thinning. Acta Ophthalmol. 2016; 94(5):e371-e372.

12. Tanito M, Sano I, Ikeda Y, Fujihara E. Short-term results of microhook ab interno trabeculotomy, a novel minimally invasive glaucoma surgery in Japanese eyes: initial case series. Acta Ophthalmol. 2017;95(5): e354-e360.

13. Tanito M, Ikeda Y, Fujihara E. Efficacy and safety of combined cataract surgery and microhook ab interno trabeculotomy in Japanese eyes with glaucoma: report of initial case series. Jpn J Ophthalmol. 2017; 61(6):457-464.

14. Tanito M. Optical coherence tomography observation of gonio structures during microhook ab interno trabeculotomy. J Ophthalmol. 2017; 2017:6310835.

15. Ahmed II. MIGS and the FDA: what's in a name? Ophthalmology. 2015;122(9):1737-1739.

16. Kahook MY, Seibold LK, SooHoo JR, Mansouri K, Sharaawy T. A nuanced approach to the surgical management of glaucoma. Middle East Afr J Ophthalmol. 2015;22(1):1.

17. Minckler DS, Baerveldt G, Alfaro MR, Francis BA. Clinical results with the Trabectome for treatment of open-angle glaucoma. Ophthalmology. 2005;112(6):962-967.

18. Malvankar-Mehta MS, Chen YN, Iordanous Y, Wang WW, Costella J, Hutnik CM. iStent as a solo procedure for glaucoma patients: a systematic review and meta-analysis. PLoS One. 2015;10(5):e0128146.

19. Khaimi MA. Canaloplasty: a minimally invasive and maximally effective glaucoma treatment. J Ophthalmol. 2015;2015:485065.

20. Matlach J, Dhillon C, Hain J, Schlunck G, Grehn F, Klink T. Trabeculectomy versus canaloplasty (TVC study) in the treatment of patients with open-angle glaucoma: a prospective randomized clinical trial. Acta Ophthalmol. 2015;93(8):753-761.

21. Seibold LK, Soohoo JR, Ammar DA, Kahook MY. Preclinical investigation of ab interno trabeculectomy using a novel dual-blade device. Am J Ophthalmol. 2013;155(3):524-529.e2.

22. SooHoo JR, Seibold LK, Kahook MY. Ab interno trabeculectomy in the adult patient. Middle East Afr J Ophthalmol. 2015;22(1):25-29.

23. Rosenquist R, Epstein D, Melamed S, Johnson M, Grant WM. Outflow resistance of enucleated human eyes at two different perfusion pressures and different extents of trabeculotomy. Curr Eye Res. 1989; 8(12):1233-1240.
Clinical Ophthalmology

\section{Publish your work in this journal}

Clinical Ophthalmology is an international, peer-reviewed journal covering all subspecialties within ophthalmology. Key topics include: Optometry; Visual science; Pharmacology and drug therapy in eye diseases; Basic Sciences; Primary and Secondary eye care; Patient Safety and Quality of Care Improvements. This journal is indexed on Submit your manuscript here: http://www.dovepress.com/clinical-ophthalmology-journal

\section{Dovepress}

PubMed Central and CAS, and is the official journal of The Society of Clinical Ophthalmology (SCO). The manuscript management system is completely online and includes a very quick and fair peer-review system, which is all easy to use. Visit http://www.dovepress.com/ testimonials.php to read real quotes from published authors. 